\title{
Théophile Gautier
}

\section{Théodore Chassériau vu par Gautier}

\section{Un document généreusement offert par la société Théophile Gautier \\ http://www.llsh.univ-savoie.fr/gautier/}

Un document produit en version numérique par Jean-Marie Tremblay, professeur de sociologie au Cégep de Chicoutimi

Courriel: jmt_sociologue@ videotron.ca

Site web: http://pages.infinit.net/sociojmt

Dans le cadre de la collection: "Les classiques des sciences sociales"

Site web: http://www.uqac.uquebec.ca/zone30/Classiques des sciences sociales/index.html

Une collection développée en collaboration avec la Bibliothèque

Paul-Émile-Boulet de l'Université du Québec à Chicoutimi

Site web: http://bibliotheque.uqac.uquebec.ca/index.htm 
Cette édition électronique a été réalisée par la Société Théophile Gautier (http://www.llsh.univ-savoie.fr/gautier/) mis en page par Frederick Diot, sous la direction de Jean-Marie Tremblay, professeur de sociologie au Cégep de Chicoutimi à partir de :

\section{Théophile Gautier Théodore Chassériau vu par Gautier}

Polices de caractères utilisée :

Pour le texte: Times, 12 points.

Édition électronique réalisée avec le traitement de textes libre OpenOffice.org 1.1 sous Linux Debian.

Édition complétée le 14 Fevrier 2004 à Bordeaux , France

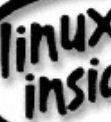




\section{La Presse, 27 mars 1844}

Le tableau le plus important du salon, pourquoi ne pas le dire tout de suite, est le Christ descendant la montagne des Oliviers, par M. Théodore Chassériau. C'est celui dont les artistes se sont tout d'abord préoccupés. Les uns l'ont trouvé admirable, les autres fort mauvais; aucun n'est resté indifférent. Ils savent bien que c'est dans ce tableau, et non ailleurs, que la question se débat. Le salon renferme des toiles qui offrent moins de prise à la critique et qui n'inquiètent personne. À coup sûr, si quelqu'un de cette génération doit devenir un grand peintre, ce sera ce jeune homme. Il y a quelques années, à propos de Suzanne au bain et d'une Vénus anadyomène nous avions tiré l'horoscope de ce talent et il ne nous a pas fait mentir. M. Th. Chassériau apporte dans la peinture un sentiment qu'on n'y avait pas encore vu et qu'on ne peut nier, qu'on l'approuve ou qu'on le blâme.

Dans notre article précédent nous avons exprimé le regret que les artistes restassent trop étrangers à la littérature, qui leur ouvrirait de nouvelles perspectives et ferait pénétrer le souffle moderne dans leurs compositions; nous n'entendons pas par là les engager à prendre des sujets dans les romans en vogue; loin de nous cette pensée; mais les poètes de ce siècle ont, dans leurs chants, révélé des côtés de l'âme humaine, compris certains aspects de la beauté, envisagé la nature sous des jours mystérieux et créé un idéal qui n'a rien de commun avec l'antique et surtout avec le classique. - Cet idéal flottant dans l'âme et dans l'esprit de tous, n'est pas représenté par la peinture, ou du moins il ne l'est que vaguement, sans conscience, presqu'à l'insu des artistes eux-mêmes, car nul ne peut se soustraire tout à fait à l'air de son temps, et les talents les plus séparés baignent par quelque coin dans l'atmosphère commune. L'équivalent de Lamartine et de Victor Hugo ne se retrouve pas parmi les peintres. Delacroix relève de lord Byron, c'est le même goût de scènes romanesques et terribles, le même amour de la Grèce et de l'Orient, le même instinct voyageur, la même passion pour les chevaux, les lions et les tigres. - S'il n'a pas été mourir à Missolonghi, il a fait le massacre de Scio. Quant à Scheffer, il est contenu presque tout entier dans la ballade de Lénore et le Faust. Marguerite lui appartient autant qu'à Wolfgang de Goethe, qui n'a jamais eu de plus intelligent traducteur. C'était en effet le temps des traductions et des poésies étrangères. La lumière venait du Nord. Ce sentiment littéraire les rendit les deux peintres les plus remarquables de cette période; ils exprimaient avec le dessin et la couleur l'idéal nouveau 
créé par l'Angleterre et l'Allemagne, et que les premiers essais de l'école romantique commençaient à répandre parmi nous. Eugène Delacroix, plus peintre que Scheffer, eut des succès contestés violemment, mais par cela même plus solides, car le champ de bataille finissait toujours par lui rester. Les artistes trouvaient, et avec raison, Scheffer trop poète et trop vaporeux ; mais le public se portait en foule à ses élégies allemandes,

Sur ces entrefaites, M. Ingres, qui depuis vingt ans produisait obscurément une foule de chefs-d'œuvre dignes des plus grands maîtres de l'Italie, et que les gens de goût de ce temps là regardaient avec dédain comme trop gothiques, fut inventé par les romantiques admirateurs des maîtres du quinzième et du seizième siècle, et porté sur le pavois précisément à cause de cette sécheresse gothique que lui reprochaient les continuateurs de David et de Girodet, incapables de comprendre autre chose que le tendon d'Achille et le nez du Jupiter Olympien. - On avait reconnu à la jeune école d'éclatantes qualités de couleur, mais on la querellait sur son dessin; car il y a des gens qui ne veulent pas comprendre qu'il y a deux sortes de dessin : - le dessin du mouvement et celui du repos. - Il fallait opposer aux classiques un homme au contour pur et sévère, $M$. Ingres se trouva sous la main, on le prit, et c'est de ce jour que datent sa gloire, sa haute position et son influence.

M. Ingres, comme tous les hommes à conviction, eut tout de suite beaucoup de partisans et d'élèves fanatiques. Il est si bon et si commode de jurer sur la parole du maître, surtout lorsque c'est un grand maître. Rien n'est plus agréable pour les esprits timorés qui ne sauraient prendre un parti de leur propre mouvement et qui se noieraient dans l'océan de leurs doutes que d'avoir un pôle invariable pour diriger leur boussole. M. Ingres, avec ses goûts exclusifs, son ton tranchant et sa peinture absolue, était plus propre que personne à rallier cette jeunesse à la débandade un peu confuse de se trouver ainsi en plein jour toute fardée et toute bariolée après ce carnaval vénitien de formes et de couleurs. À cette rude école, les jeunes élèves apprirent à connaître le véritable antique, le goût du beau dessin et du grand style; la sobriété des moyens d'exécution les força à chercher les qualités sévères; et à défaut de coloris, ils acquirent une harmonie relative, une finesse de modelé, une douceur d'aspect supérieures, à notre avis, à ces tapages de nuances criardes que les Français ne sont que trop disposés à prendre pour de la couleur. Ziegler, Lehmann, Flandrin, Amaury Duval et Chassériau, pour ne parler que des plus importants, sortirent de cet atelier austère avec un respect et une terreur du maître qui ne sont pas encore dissipés chez quelques uns. Théodore Chassériau secoua l'un des premiers le joug: tout en restant 
fidèle à certaines habitudes de dessin, à certaines teintes grises, il commença sous une grande placidité d'aspect à se livrer à des turbulences de composition, à des jets de draperies, à des audaces de mouvement et de style qui ne sentaient déjà plus l'écolier; dans ses tableaux, les yeux, jusque là imités des yeux à regards blancs des marbres grecs, commencèrent à jeter des lueurs étranges, les bouches arquées par ce froid dédain de la vie qui caractérise $M$. Ingres, s'entrouvrirent comme si elles voulaient murmurer quelque chose; les bras se séparèrent des corps, comme cela dut avoir lieu lorsque les sculpteurs égyptiens, las de tailler des figures hiératiques, dégagèrent du bloc les membres de leurs statues; le contour prit de la liberté, et la touche une fierté magistrale. Les Femmes troyennes au bord de la mer, l'Andromède au rocher, quoique conçues dans le goût antique le plus pur, ont un cachet particulier, un aspect bizarre et saisissant, - c'est la différence d'un dessin de vase étrusque brun et rouge, à la blancheur placide d'un bas relief du Parthénon; dans les têtes respirait une mélancolie mystérieuse, une sauvagerie primitive, les regards noirs de toute l'ombre du passé, avaient une expression singulière dans leurs masques de marbre pâle. C'était du grec, ou plutôt du pélage du temps d'Orphée.

À la même place, à peu près, où se trouve aujourd'hui le Christ descendant du jardin des Oliviers, M. Chassériau a exposé, si nous avons bonne mémoire, un Christ recevant des mains de l'archange le calice rempli jusqu'au bord de l'âcre vin des douleurs. Le tableau dont nous allons parler est comme le pendant et le complément de l'autre, et quoique d'une composition très simple et d'une action tranquille, il est empreint d'une désolation et d'une tristesse plus profondes encore.

Il fait nuit ; la veille d'agonie est terminée; le Christ, après ces terribles épreuves, ces sueurs de défaillance, ces angoisses pendant lesquelles des doutes lui sont venus sur sa divinité, redescend, la coupe vidée, le penchant de l'âpre colline, d'un pas brisé, les bras morts, la tête flottante sur la poitrine, dans un état de prostration complète; les oliviers centenaires tordent leurs troncs difformes et tendent vers le ciel, comme des bras suppliants, leurs moignons mutilés; leurs racines s'enfoncent hideusement, comme des reptiles en fuite, dans les interstices des rochers, et la lune tamise sa lueur livide à travers les feuillages glauques et déchiquetés. Le chemin, d'une blancheur poussiéreuse, rayé par les ornières des chars, rampe tristement au flanc du monticule. Sur le revers de la route sont étendus les trois apôtres, Pierre, Jacques et Jean, buvant à pleines gorgées dans la noire coupe du sommeil ; et pourtant le maître leur avait dit : «Mon âme est triste jusqu'à la mort; attendez moi ici et veillez. 
»C'est peut être là le moment le plus triste et le plus douloureux de la passion du Christ. Se dévouer pour quelqu'un qui s'endort pendant votre supplice ! Pierre et Jacques, passe encore: ce sont des gens d'action, des apôtres rustiques et populaires; ils étaient sans doute fatigués de leur journée. - Mais toi, jeune homme à la figure et aux cheveux de femme, toi, nature délicate et sensible, toi, le disciple bien aimé, les larmes n'ont donc pas pu tenir tes paupières ouvertes ! et bientôt vaincue, ta tête a roulé sur ton bras nonchalant! Ah ! Jean, quel remords lorsque tu seras éveillé !

Le Christ, debout derrière ce groupe de dormeurs, les considère mélancoliquement d'un air affligé, mais non surpris : il s'attendait à cela; il connaissait la faiblesse humaine, et savait combien sont vaines les protestations de foi et de dévouement, lui qui dit à saint Pierre: «Avant que le coq ait chanté, vous m'aurez renié trois fois. »

Les trois dormeurs sont très beaux, surtout le saint Jean; leurs poses, quoique naturelles, se font remarquer par une noblesse qui n'exclut pas l'abandon; les draperies s'agencent parfaitement, quoique serrant le nu d'un peu trop près. La tête du Christ est magnifique d'expression et d'exécution. Dans un coin du tableau, une lueur de torches fait deviner les satellites, qui s'avancent, conduits par Judas. Mais qu'importe le baiser du traître à barbe rousse ? Jean ne s'est il pas endormi ! Le Christ n'a t il pas été déjà blessé à l'endroit le plus secret et le plus tendre de son cœur!

Le fer de Longus ne pénétrera pas plus avant dans la poitrine du divin martyr.

Certes, ce tableau représente très exactement la scène indiquée par l'évangéliste Saint Matthieu. Ce sont bien là Jésus de Nazareth, Pierre, Jacques et Jean; les ajustements, les costumes sont empreints du goût antique, mais une douleur moderne palpite sous la tunique traditionnelle du Christ; ce sont les larmes de notre temps qui coulent par ces yeux, notre mélancolie s'épanche dans ces cheveux en pleurs; cette figure intelligente et fatiguée n'a aucun rapport avec les images byzantines, aux regards impassibles, ni avec les longues effigies gothiques. C'est la souffrance inquiète de notre âge qui a martelé ces joues et bleui ces orbites; ces mains fluettes ont la pâle maigreur des mains de poète, et les clous des bourreaux n'y rencontreront que des veines et des nerfs! Sans vouloir prêter ici au peintre plus d'intentions qu'il n'en a, ne peut on pas voir ici un symbole général du sacrifice et de l'indifférence ? - Ce Christ et ces dormeurs ne signifient ils pas l'âme et le corps? Le poète qui descend des hauts sommets de l'inspiration après une de ces entrevues avec Dieu qui font 
monter aux tempes des sueurs de sang, et qui trouve au bas de la montagne ses disciples les plus chers, ses auditeurs les plus assidus perdus dans le sommeil des brutes; l'être dévoué qui a offert et donné sa vie pour ceux qu'il aime et qui, en marchant à l'échafaud, les rencontre distraits et froids, et n'ayant plus l'air de le connaître. Dans cette toile désespérée respire quelque chose du sentiment que $\mathrm{M}$. de Lamartine a mis dans ses novissima verba; on devine dans le regard morne et pensif du Christ que si le sacrifice était encore à faire, il ne le recommencerait pas. Il est malheureux que cette toile n'ait pas été accrochée plus bas. Quoique de grande dimension, elle perd à être vue de si loin. - On ne peut pas aussi bien apprécier la finesse du pinceau et le mérite des détails. Il faut espérer que lorsqu' on changera les cadres de place, à la fermeture temporaire du Musée, le Christ descendant du jardin des Oliviers ne descendra pas de si haut.

M. Th. Chassériau avait commencé une Mort de Cléopâtre qu'il n'a pu terminer. Cela n'a rien d'étonnant, lorsqu'on songe aux importantes peintures murales qu'il a exécutées dans la chapelle de Sainte Marie l'Égyptienne, et dont nous avons rendu compte, nos lecteurs s'en souviennent peut être, il y a quelques mois, dans la Presse. Il faut toute la fougue de travail qui caractérise ce jeune peintre, pour avoir trouvé le temps de paraître au salon avec une composition de cette importance. Nous ne saurions trop l'en féliciter. L'artiste ne saurait que gagner a communier fréquemment avec le public. Il donne à la foule, mais la foule lui rend.

\section{La Presse, 25 mai 1832}

Il nous semble qu'il nous arrive un malheur personnel lorsqu'un artiste que nous aimons, et en qui nous avions foi, ne tient pas ce que nous étions en droit d'espérer de lui : soit qu'il perde son talent, soit qu'il s'interrompe dans sa marche, pour une raison ou pour une autre. Certes, si nous avions jamais éprouvé une vive sympathie pour quelqu'un, c'est assurément pour Théodore Chassériau. Lié avec lui depuis de longues années, nous l'avons fraternellement suivi depuis ses premiers essais, nous plaisant à voir le développement de cette intelligence supérieure pour laquelle nulle ambition n'eût semblé trop haute. Chacun de ses travaux a été, de notre part, l'objet d'une étude étendue, d'une analyse consciencieuse, 
quoiqu'elle ait pu paraître passionnée à des gens moins convaincus que nous du génie du peintre ; nous regardions alors et nous regardons encore Chassériau comme une des natures les mieux douées de notre époque, malgré l'usage regrettable qu'il a fait depuis quelque temps de ses merveilleuses qualités. Chassériau, formé sous la rude discipline de l'école d'Ingres, et longtemps l'élève chéri de ce maître austère, est dessinateur par nature, par éducation et par goût. Ses dons sont la ligne, le style, le caractère, la noblesse. Nourri aux plus pures doctrines grecques, il avait un sentiment de l'antique pour ainsi dire contemporain; il était né étrusque, comme d'autres le sont devenus à force d'abstraction, et vivait sans pastiche dans cette grande Grèce dont Herculanum, Pompeï et les vases de Nola ont trahi les secrets. À la pure beauté de Phidias, à l'élégance athénienne, il joignait un sentiment mystérieux et triste, une certaine grâce sauvage, une indéfinissable langueur orientale. A ses masques de marbre, il mettait souvent des yeux de sphinx pleins d'énigmes et de rêverie, d'une douceur profonde, d'une fixité inquiétante et sereine; ses bouches, un peu plus épanouies que les bouches classiques des statues, semblaient aspirer un tiède souffle d'Orient, et quelques coquetteries barbares d'ajustement, quelques bijoux exotiques indiquaient le voisinage de l'Asie et de l'Égypte. On eût dit un élève d'Apelle ayant suivi Alexandre à la conquête des Indes, et mêlant, à son retour en Grèce, quelques souvenirs des races étrangères aux purs types de son pays. Cette assimilation rétrospective, que nous avons déjà faite quelque part, représente on ne peut mieux, selon nous, la nature de talent du jeune artiste dans toute son originalité primitive. Il fit, sous cet ordre d'idées, une Suzanne au bain conçue au point de vue antique oriental et d'un type poétiquement retrouvé, que nous considérions comme une de ses meilleures choses. La beauté israélite ennoblie par l'art de la Grèce ne fut peut être jamais réalisée avec plus de bonheur : une sorte de pudeur biblique revêt la chaste nudité de ce beau corps épié par les obscènes vieillards, accroupis dans l'ombre comme des bêtes fauves prêtes à sauter d'un bond sur leur proie. Ce torse parfait, que la Vénus de Milo ne désavouerait pas, par la coupe plus aiguë du sein, par la sveltesse plus évidée du flanc, se rattache aux formes de l'Orient et rappelle vaguement les prêtresses de l'Inde, desservantes des pagodes, qui descendent au Gange par les escaliers de marbre blanc de Bénarès; la tête a une smorfia mélancoliquement dédaigneuse, une expression langoureuse et virginale dont le souvenir vous poursuit ; la Vénus sortant de la mer et tordant les perles de sa chevelure blonde, entre l'azur de la mer et l'azur du ciel, nous donne l'idée de ce que pouvaient être les tableaux de ces merveilleux peintres grecs, dont Pline a écrit l'histoire, et dont les oeuvres, dévorées par les siècles jaloux, ne nous sont pas parvenues. Figurez vous un marbre de Paros, légèrement teinté comme l'étaient les statues 
antiques. Les Troyennes captives, pleurant au bord de la mer, avaient une mélancolie toute virgilienne, et traduisaient admirablement le pontum adspectabant flentes, ce demi vers grand, plaintif et triste comme l'immensité bleue qu'il est destiné à peindre; leur douleur ne dérangeait pas leur beauté ; leur désespoir féminin pleurait avec grâce et s'étageait en groupes charmants sur le rivage et les rochers. À ce cycle greco asiatique se rattache une Andromède exposée, une Diane surprise, un Apollon poursuivant Daphné, d'une mythologie renouvelée et puisée à la source même; - puis vinrent les peintures de la chapelle de Sainte Marie l'Égyptienne à Saint-Merry, sujet admirablement approprié au talent du peintre, et qu'il traita d'une façon supérieure dans ce goût demi grec, demi barbare, avec cette beauté étrange et farouche qui caractérisaient sa première manière; - il fit aussi quelques tableaux de sainteté, un Christ descendant $d u$ jardin des Oliviers et trouvant ses disciples endormis; jusque là, l'originalité de l'artiste était restée intacte: il marchait dans sa force et sa liberté, exalté par les uns, dénigré par les autres; loué ou critiqué violemment comme tout homme d'une vraie valeur, il ne relevait que de lui, sauf ces ressemblances éloignées de l'élève au maître, qui sont aussi honorables que celle du fils au père, et prouvent seulement qu'on est de bonne race.

Arrivé là, nous ne savons sous quel revirement intérieur notre artiste se troubla; il adora ce qu'il avait brûlé et brûla ce qu'il avait adoré; une influence, celle que l'on devait assurément le moins redouter pour lui, le fit dévier dans son talent déjà formé et constaté par des oeuvres nombreuses. Delacroix inspira au jeune maître nous ne savons quelle émulation funeste, et lui donna le vertige du mouvement et de la couleur. Il voulut le combattre, non pas avec son casque hellénique à crinière rouge, son bouclier de cuir de taureau plaqué d'étain, son glaive de bronze et ses knémides d'airain, mais avec la dague de Tolède, l'armure de Milan, le kandjar turc et toute la panoplie romantique. Il prit les armes de son adversaire et jeta les siennes qu'il excellait à manier. - Lui, champion du dessin, il attaqua Delacroix sur le terrain de la couleur. Aveuglement étrange! Il cessa de lire la Bible, Homère, Hésiode, Virgile, et se mit à feuilleter Shakespeare, Goëthe et les modernes ; il alla avec les autres au grand sabbat sur le Brocken, au lieu de rester à Larisse et d'épier au clair de lune les belles magiciennes de Thessalie.

Les peintures de l'escalier de la Cour des comptes, au quai d'Orsay, montrent, comme des fleuves dont les eaux ne sont pas encore mélangées, les deux manières de l'auteur, l'ancienne et la nouvelle, se côtoyant sans se confondre encore, l'ancienne claire et calme, la nouvelle orageuse et 
trouble; le côté de muraille qui représente la paix entourée du groupe tranquille des arts et des industries rustiques, appartient à Chassériau; l'autre sujet, symbolisant la guerre, revient à Delacroix par l'inspiration.

Un voyage en Algérie entrepris vers ce temps-là aggrava la maladie pittoresque de l'artiste. L'Orient est dangereux, surtout l'Orient barbaresque; il fait naître un vertige que nous concevons très bien, l'ayant éprouvé par nous même. Au milieu de nos civilisations effacées, cela produit l'effet d'un mardi gras en carême, d'un carnaval en plein soleil et d'une mise en scène d'opéra dont l'auteur ne veut pas se nommer: ces vestes brodées, ces ceintures hérissées d'armes, ces selles bosselées d'or, ces longs fusils ornés de corail, ces burnous blancs aux plis majestueux, ces chevaux ardents et maigres, aux narines roses, aux crinières teintes de henné, tout cela trouble et jette dans une ivresse bizarre. Peu de ceux qui ont $\mathrm{vu}$ ce spectacle, même parmi les plus robustes, y résistent complètement; chacun en revient un peu musulman dans son cœur, et il arrive parfois en pays chrétien, lorsqu'il pleut et que les bourgeois sont trop laids dans la crotte, de penser aux minarets de Constantine, aux fantaisies des douairs arabes, et de dire à mi voix: il n’y a de Dieu que Dieu, et Mahomet est son prophète. Chassériau fut impressionné très vivement par ces beaux types, ces costumes superbes, cette âpre et forte nature, comme le prouvèrent son Sabbat Juif à Constantine, son Kaid visitant les tribus, ses Cavaliers ramassant des morts, sa Mêlée et autres épisodes de la vie arabe; mais, cette fois, il ne s'abandonna pas à son sentiment particulier et n'aperçut pas cette nature au point de vue original qu'on attendait de lui: les Consulsionnistes de Tanger, les Femmes d'Alger, l'Empereur du Maroc, la Noce juive, toute la galerie barbare d'Eugène Delacroix resta trop présente à sa mémoire, et cependant il y avait en Afrique pour Chassériau un côté complètement neuf que Delacroix ni personne n'y a vu, nous ignorons pourquoi, car il est très discernable, et un peintre imbu du sentiment antique devait le démêler tout de suite : c'est la beauté, le style, la grandeur sculpturale, la noblesse historique des Arabes. Decamps et Delacroix en ont trop souvent fait des macaques et des mandrils. Dans chaque figure orientale peinte par ces artistes, il y a toujours un singe caché; quelquefois le singe se tient tranquille et debout sur ses pattes de derrière, mais à la première occasion, il se gratte l'aisselle, croque un pou, fait une grimace et une gambade. Pourquoi Chassériau, qui sait dessiner comme un ancien élève d'Ingres qu'il est, au lieu de colorier bizarrement des bonshommes, n'a-t-il pas rendu avec une sévérité antique ces types aussi purs, aussi nobles que ceux des statues de Phidias, ces superbes draperies qu'on croirait sculptées dans le marbre du pentélique, ces formes sveltes, élégantes et vigoureuses, ces 
yeux si limpides, ces nez si droits, ces bouches d'une coupe si nette, ces cavaliers robustes et nerveux, ces jeunes gens aussi beaux que des vierges de Raphaël et qui feraient croire a un troisième sexe, ces femmes entrevues en qui revivent la reine de Saba, Rachel, Nourmahal et tous les mirages de la Bible et des Mille et une Nuits ? - jusqu'à présent, on n'a guère vu que la friperie de l'Orient; les costumes ont distrait des figures. Sous ce rapport, c'est une mine à peine effleurée, on y a seulement pris à la hâte quelques poignées de rubis et de diamans que l'on ne s'est pas donné la peine de tailler. Dans ce monde nouveau, il y a plus à faire encore pour la ligne que pour la couleur. Nous aurions voulu voir notre ami peindre l'Orient dans ce sens ; il s'y fût montré complètement neuf et avec toutes ses ressources.

Sans doute, dans la plupart des oeuvres de Chassériau que nous venons de citer, il y a un talent incontestable et des qualités de premier ordre; nous déplorons seulement que l'artiste, qui pouvait vivre magnifiquement chez lui, dans son palais de marbre aux colonnes ioniennes, aille sonner de l'olifant devant le castel moyen âge d'Eugène Delacroix.

Nous insistons beaucoup sur ceci, au risque de froisser peut être une amitié ancienne et précieuse, parce que cette crise se renouvelle chez presque tous les peintres au commencement de leur seconde manière : ou ils s'abjurent ou se jettent dans quelque imitation contraire, et Chassériau nous parait se tromper complètement de route, surtout si l'on doit considérer comme l'expression exacte et actuelle de son talent les trois tableaux de nature diverse qu'il a exposés cette année, et qui, chacun, exprime une déviation de ce que nous croyons, après mûr examen, être son individualité primitive.

Ses Cavaliers arabes, s'injuriant avant le combat à la façon homérique, auraient prêté à un développement de nobles formes, d'attitudes superbes, de draperies au grand jet que l'artiste a négligé pour une turbulence d'esquisse, une furie de brosse qui montrent la préoccupation d'une autre manière; des incorrections voulues, une touche heurtée, des oppositions violentes de ton, donnent à cette toile l'aspect d'une ébauche plutôt que d'un tableau ; les types des têtes sont laids et ont dû coûter bien de la peine au peintre élégant et pur de la Vénus sortant de l'écume, qui n'aurait qu'à laisser courir son pinceau pour produire de belles figures d'une originalité parfaite.

Le Jésus chez. Marthe et Marie offre aussi la même tendance à chercher le caractère aux dépens de la beauté, la couleur aux dépens de la sévérité du 
dessin. La tête du Christ manque de noblesse et n'a pas cette mélancolie divine que l'artiste a su lui donner à une époque plus heureuse. Ses extrémités sont négligées et communes; la figure de Marthe, d'une jolie intention et d'une tournure originale, est indiquée trop vaguement, et la tête de Marthe qui écoute dans une inaction passionnée la parole du Sauveur, tandis que sa sœur s'agite et vaque aux soins domestiques, rappelle seule l'ancien Chassériau : elle est noble et pure et possède un charme féminin très rare aujourd'hui chez les artistes sérieux et dont Eugène Delacroix, entr'autres, est complètement dénué.

Dans sa Desdémone se faisant déshabiller par sa suivante, le peintre reprend, en le modifiant un peu, un thème qu'il a déjà traité sous forme d'eau forte en illustrant l'Othello de Shakspeare. Le motif a une sorte d'élégance sculpturale tout à fait dans la nature de talent de l'artiste. Cette belle jeune femme, agitée de pressentimens funèbres, pâle comme la statue d'albâtre de son tombeau, et quittant ses voiles blancs pour se coucher dans ce lit d'où elle ne se relèvera plus, est, en effet, un beau sujet digne des efforts de l'art le plus élevé. Malheureusement le peintre ne s'est pas donné la peine de l'exécuter après en avoir trouvé heureusement la disposition et les lignes principales. À quoi bon ce faire inculte, cette brosse rude, ces lourds empâtemens dans un tableau de chevalet dont les figures ont quinze pouces de hauteur? Pourquoi rendre grossier par l'exécution ce qui est délicat par l'idée et la forme? Un cadre d'un pied carré ne peut pas être traité en peinture murale.

Nous avons vu dans l'atelier du peintre un tableau qu'il n'a pas achevé, un tepidarium de bain antique à Pompeï ; le tepidarium est, dans les thermes, la salle tiède où l'on fait boire aux linges velus la dernière perle d'eau ou de sueur, où l'on se familiarise avec l'air respirable au sortir de la brûlante étuve; c'est un lieu de causerie nonchalante ou de repos rêveur, où l'on flâne délicieusement avant de reprendre ses habits. - Les bains mores d'Alger vous donnent une idée de ce que pouvait être le tepidarium de Pompeï, qui, du reste, subsiste encore presque intact avec sa corniche de petits Hercules de terre culte, formant des niches au dessus de la tête de chaque baigneur pour serrer ses vêtements. - Une douzaine de jeunes femmes réunissant les types chers à l'artiste, rappelé cette fois forcément à l'antiquité et à ses études primitives, sont isolées ou groupées dans des poses pleines d'élégance et de naturel : on dirait les fresques de la maison de Salluste ou du poète tragique qui se sont détachées de leurs murailles et revivent de leur vie familière.

Ce tableau nous a paru un symptôme de convalescence, un retour de santé 
artistique. L'antiquité est saine, et, quand il y revient, guérit l'art de bien des maladies. Espérons que Chassériau va bientôt, débarrassé de toute idée étrangère, rentrer en maître dans sa propre originalité. - La seconde manière d'un peintre ne doit pas être l'opposé de la première, mais seulement sa nature développée, agrandie, rendue plus robuste par l'âge, le travail et l'expérience. - C'est une spirale qui s'ajoute à l'autre montant plus haut mais ayant le même point de départ.

\section{La Presse, 24 juin 1853}

Nous avons éprouvé, en voyant le Tepidarium de Théodore Chassériau, une des plus vives satisfactions de notre vie de critique, un sentiment pareil à celui que cause la convalescence et le retour à la santé d'un ami qu'on avait cru perdu. Déjà nous avions déploré en nous même la maladie de ce beau talent, maladie qui semblait être mortelle, et chanté tristement la nénie de ce charmant esprit égaré. Au risque de faire à une amitié ancienne une de ces blessures qui se cicatrisent si difficilement, notre voix l'avait adjuré de se souvenir de lui et de ne pas compromettre, par des imitations étrangères, une des originalités les plus nettes de ce temps ci. Nous le sommions ici lui-même de retourner à son corps qu'il quittait, comme ce brahme indien, pour faire des voyages dans l'individualité des autres, lui rappelant qu'il pourrait bien plus tard le retrouver déchiré par les chacals et les oiseaux de proie; en effet, nulle peau ne nous gante mieux que la nôtre, et la peau d'un berger l'habille plus juste que ne le ferait la peau d'un roi écorché.

Cette année (nous n'avons pas l'orgueil d'attribuer ce changement heureux à nos conseils), l'artiste, redevenu maître de lui même et rejetant toute pourpre d'emprunt, est descendu dans l'arène nu et frotté d'huile comme un lutteur antique. Il a essuyé le fard de ses joues, et reparaît avec sa tranquille pâleur de marbre ; l'ivresse des coupes vertigineuses est passée, et il revient éprouvé et sûr désormais au culte de la beauté sereine, l'amour si bien inspiré de sa première jeunesse. - Certes, pour une âme vouée comme la nôtre aux adorations de l'art, il n'est pas de volupté plus ineffable que de voir une de ses admirations mortes se relever du tombeau en entrouvrant son blanc linceul. C'est comme Roméo voyant, miracle inespéré, Juliette se redresser sous la voûte du caveau de Vérone. Il est si triste de ne plus admirer le talent qui réalisait votre secret idéal, de 
renverser la statue du socle où soi même on l'a élevée !

Le Tepidarium de Chassériau est un des plus remarquables tableaux de l'exposition de 1853. Il reluit comme un joyau enchâssé à la plus belle place du salon carré, la tribune de ce Louvre annuel, et c'est justice.

En dix huit cent cinquante, nous visitions Pompeï, - ce palimpseste de la civilisation antique si merveilleusement remis au jour, et transporté à deux mille ans en arrière au milieu du monde greco romain, nous parcourions d'un pas respectueux, pour ne pas soulever la poussière des siècles, les ruines des Thermes, encore assez bien conservées pour qu'on puisse les restaurer facilement par la pensée et retrouver l'art balnéatoire des anciens, si compliqué et si parfait à côté de nos insuffisans lavages.

Nous étions parvenu dans une salle charmante, à la voûte presque intacte, entourée d'une série d'Hercules ou d'Atlas en terre cuite supportant une corniche richement ornementée et formant, entre leurs interstices, une suite d'armoires ou de cabinets pour serrer les habits; quelques vestiges de calorifères et de fontaines se distinguaient le long des murs et sur le pavé ; une ouverture de la voûte laissait briller un coin de ciel tendrement bleu et d'une transparence profonde; nous restâmes là longtemps, refaisant le pavé de mosaïque, restituant les moulures brisées, remettant en place les marbres abolis, repeuplant le Tepidarium désert avec les jeunes femmes de Pompeï, dont il ne reste maintenant qu'un bracelet d'or ou qu'une boucle d'oreille de perle dans une pincée de cendre; nous coloriions les statues du musée de Naples, nous détachions les fresques de la maison de Salluste et $\mathrm{du}$ poète tragique pour les douer d'une vie fantasmatique et les faire asseoir ou se coucher autour de la vaste salle en des attitudes de grâce et de nonchalance, si préoccupé d'ailleurs de notre rêve, que nous en perdîmes nos compagnons.

Malheureusement, la traduction pure et simple de la beauté qui suffit au peintre ne suffit pas au poète, et notre songe rétrospectif s'évanouit sans laisser de trace. Jugez de notre enchantement lorsque nous avons vu exécuté par un pinceau habile le tableau dont l'idée nous était venue dans le Tepidarium de Pompeï et qui, par un magnétisme secret, s'était présentée aussi à l'imagination de notre ami Théodore Chassériau, redevenu un Grec pur après s'être mêlé quelque temps aux cohortes des barbares.

La salle que nous venons de décrire sert de fond aux figures groupées par Chassériau ; seulement, à la place des Hercules de terre cuite, il a substitué 
des statuettes de bronze, et revêtu de plaques de brèche rouge les cabinets à serrer les vêtements. Une guirlande sculptée joue aux parois de la voûte, et le ciel bleuit au delà de l'étroite fenêtre avec une illusion de diorama. À partir du cadre, dont les lignes d'or le séparent des réalités environnantes comme la rampe trace une démarcation de feu entre la scène et le théâtre, le Tepidarium s'enfonce dans la muraille et produit par la perspective une étonnante illusion, le trompe-l'œil le plus parfait. C'est là, nous le savons, un mérite secondaire, mais lorsqu'il ne nuit en rien à la sévérité du style, qu'il n'est pas obtenu par des sacrifices nuisibles aux sérieuses qualités de l'art, il ne peut qu'ajouter du charme et de la valeur à la composition.

Au premier plan, une jeune femme, debout, le torse nu, et dont la cuisse un peu fléchie retient une draperie près de glisser, étire ses bras avec ce mout vement de bien être indolent et cette sorte de spasme voluptueux qui suit les délicieuses lassitudes du bain; elle fait ressortir ainsi les belles lignes et les richesses juvéniles de son corps rosé et moite des tiédeurs de l'étuve; la lumière amoureuse glisse par larges nappes sur ces formes pures, sur ces chairs fermes et souples qui n'auraient qu'à se décolorer légèrement pour se transformer et marbre de Paros. Le peintre a caressé con amore cette figure, le diamant d'un écrin de beautés, celle que l'œil rencontre d'abord au centre du tableau et qui le retient longtemps avant de le laisser s'égarer parmi les autres baigneuses toutes diversement charmantes; elle est en quelque sorte le camée de ce bracelet de femmes.

Près d'elle, sur un escabeau d'ivoire, est assise une jeune Pompeïenne déjà demi vêtue d'une draperie rose et dont la tunique jaune traîne sur les dalles luisantes; un coin d'épaule sort de ses linges, pur et brillant comme le disque de la lune du pli d'un nuage. Un peu plus loin, vers l'angle du tableau, une autre femme à la tête patricienne, à la beauté noble et sévère, se drape comme une Junon dans l'ampleur d'un grand manteau blanc; à l'autre angle, une baigneuse nonchalamment entourée de ses draperies, qu'elle arrête d'une main négligente, va rejoindre le groupe de ses compagnes, montrant au spectateur un dos d'une couleur et d'un modelé superbes, une nuque blondissante où pétillent dans la lumière des flocons de cheveux d'or.

Au delà commence une foule bigarrée et charmante de types antiques puisés aux sources les plus pures ou plutôt déjà vus par l'intuition d'un génie particulier; autour d'un grand brasero de bronze portant sur des pattes de lion, une rangée de femmes plus ou moins nues ou plus ou moins habillées tendent leurs mains à la chaleur de la flamme dont le reflet les éclaire, se rajustent, se sèchent, font boire à des étoffes moelleuses les 
dernières perles du bain ou se reposent tout simplement; les unes rêvent, les autres causent, et derrière elles circulent les esclaves apportant les boîtes de parfums, les cassettes à bijoux, les miroirs de métal ou quelque coupe d'argile ou d'or pleine d'une boisson frappée de neige; une Éthiopienne aux tons fauves, une Gauloise à chevelure rousse cherchent, dans une de ces niches séparées par des statues, la tunique, la chlamyde ou le peplum de leur maîtresse.

Une chose nous frappe surtout dans le talent de Théodore Chassériau, c'est à quel point il est naturellement antique. Ce n'est pas dans l'étude des statues, des médailles, des fresques, des vases étrusques qu'il a puisé ce grand goût grec que personne ne possède à un si haut degré que lui; il ne fait pas d'archaïsme et ne reconstruit pas laborieusement le passé; ce passé, il le porte en lui jeune et vivant. Tout cet art disparu ou resté à l'état d'idéal existe virtuellement pour lui; - il a les yeux d'un peintre de l'école de Sycione ou d'Athènes, et tracerait sur les marbres de la Pinacothèque des Propylées, à la place où étaient les tableaux de Polygnote, des figures d'un style qui ne jurerait pas à côté des Métopes et des Panathénées de Phidias, et c'est pour cela que nous regrettions amèrement de le voir s'engager dans d'autres voies, parce que peu de gens ont ce privilège d'être, au dix neuvième siècle, un Grec naïf du temps de Périclès,

Le seul côté moderne qu'aient les têtes de Chassériau, ce sont les yeux empreints d'une fixité rêveuse ou noyés d'une langueur nostalgique absentes du regard blanc des statues; ces figures, d'une sérénité morne et d'une passivité dédaigneuse, rappellent les belles esclaves grecques captives à la cour de quelque roi barbare qui les adore et qu'elles méprisent tout en subissant son amour, par exemple Myrrha, dans le palais de Sardanapale ; elles regrettent, sans en avoir la conscience peut être, les rochers de marbre de l'Attique, les lauriers roses du Cephise, les pins sombres du Parnès et le triangle neigeux du Parthénon se détachant sur le fond d'améthyste du Pentelique et du Lycabète. Cela les ennuie de se voir entourées des vulgarités de la vie moderne, elles faites pour porter le van aux fêtes d'Eleusis ou se dérouler en théorie sur le couronnement d'un temple.

Le seul reproche que nous ferons à l'artiste est d'avoir négligé quelques extrémités et parfois attaqué d'une brosse trop rude certaines délicates portions de nu. Nous ne demandons pas un poli excessif, mais il ne faut pas que la gradine raie trop violemment la chair immortelle du Paros; une exécution tranquille convient à la beauté suprême, et toute trace de l'outil, c'est à dire du moyen matériel, doit disparaître dans un chef-d'œuvre où 
tous les détails antiques sont d'une exactitude extrême: le brasero, les banquettes de bronze existent au musée de Naples, car maintenant les brillans anachronismes de Paul Véronèse ne sauraient plus être excusés.

Le Mazeppa recueilli par une jeune fille de l'Ukraine est un charmant petit tableau d'une importance moindre, sans doute, que le Tepidarium, mais qui vaut qu'on s'y arrête. Le cheval mort s'est abattu sur le flanc, renouvelant le supplice de Mézence, et retenant lié à son cadavre un corps vivant. Mazeppa, qui n'a plus la force de se tordre dans ses nœuds, gît, inerte, bleuâtre et glacé, ne sachant pas que, selon la magnifique expression du poète, il va se relever roi. Au delà, la steppe s'étend indéfinie, et le cercle des corbeaux tourne dans l'air avant de s'abattre sur sa proie. La jeune Cosaque s'avance avec une curiosité craintive mêlée de pitié. Sa tête est délicieuse. Théodore Chassériau excelle à rendre ces types mystérieux des races inconnues; il leur donne une grâce sauvage, une coquetterie bizarre, dont le charme se sent plus qu'il ne s'explique. 